\title{
A Pictorial Review on the Role of 64-Slice HD MDCT in Detecting Post CABG Cardiothoracic Complications
}

\author{
Ibrahim M. Helmy', Akram M. Asbeutah' ${ }^{2}$, Inas M. ElFiki ${ }^{3}$, Osama E. Arafa ${ }^{4}$ \\ ${ }^{1}$ Department of Clinical Radiology, Co-Operative Union Cardiac Hospital, Ministry of Health, Kuwait City, Kuwait \\ ${ }^{2}$ Department of Radiologic Sciences, Faculty of Allied Health Sciences, Kuwait University, Kuwait City, Kuwait \\ ${ }^{3}$ Radiology Department, Zagazig University, Zagazig, Eygpt \\ ${ }^{4}$ Department of Cardiothoracic Surgery, Co-Operative Union Cardiac Hospital, Ministry of Health, Kuwait City, Kuwait \\ Email: asbeutah akram@hotmail.com, akram.asbeutah@hsc.edu.kw
}

Received 2 May 2014; revised 2 June 2014; accepted 20 June 2014

Copyright (C) 2014 by authors and Scientific Research Publishing Inc.

This work is licensed under the Creative Commons Attribution International License (CC BY). http://creativecommons.org/licenses/by/4.0/

(c) (i) Open Access

\begin{abstract}
Aims: The aim of this study is to have a pictorial review on the role of 64-slice multi-detector computerized tomography (MDCT) in detecting post coronary artery graft (GABG) cardiothoracic complications. Materials \& Methods: During the period from November 2012 to December 2013, a prospective evaluation of 40 patients with suspected post-operative cardiothoracic complications underwent MDCT coronary angiography in our clinical radiology department. Informed consent was obtained from all patients and the study had institutional review board approval. Descriptive statistics were used to analyze the data. Results: There were 35 males and 5 females, their mean age \pm (SD) of $57 \pm 3.9$ years. A total of 60 grafts were available for evaluation. Two patients could not be evaluated due to clip-artifacts and advanced atherosclerotic disease. Eleven patients showed occluded graft (9) at proximal and distal (2) anastomosis. Seven patients showed pericardial effusion and pleural effusion (8). Two patients had chest wall infection with retrosternal extension and associated mediastinal involvement. One patient showed aneurysm at the re-implanted coronary artery after Bentall procedure, another patient had postoperative myocardial infarction, one patient showed dissection of the ascending aorta, one patient pseudoaneurysm of left ventricle, two patients localized pericardial hematoma, two patients pulmonary embolism, and two patients showed pneumonia. All these complications occurred from 1 - 60 days from surgery. Conclusion: HD MDCT is an easy non-invasive technique which showed to be effective in diagnosis of most postoperative CABG cardiothoracic complications and is gaining more ground in difficult clinical situations.
\end{abstract}

\section{Keywords}

Cardiothoracic Surgery, Cardiothoracic Complications, Multi-Detector Computerized Tomography, 


\section{Coronary Arterial Bypass Graft}

\section{Introduction}

Over the last decade, advances in computed tomography (CT) technology have revolutionized the diagnosis of cardiovascular disease, reduced the need for diagnostic arterial catheterization, and became an invaluable tool in the diagnosis and pre operative evaluation of coronary disease [1]. Moreover, Multidetector CT (MDCT), introduced into clinical practice in 2000, has demonstrated excellent detection of significant coronary lesions with a high degree of diagnostic accuracy and showed excellent capability of excluding coronary lesions, due to high negative predictive values, which ranges from $96 \%$ to $99 \%$ [2].

Complications of open heart surgery for coronary artery disease (CAD) can be categorized into two groups: graft related and non-graft related surgical complications. The graft related surgical complications of coronary arteries include spasm [3], graft thrombosis [4] [5], malposition, kinking [5] [6], and iatrogenic complications such as retained clips, aneurysm and pseudoaneurysm of graft and ascending aorta, ostium stenosis and occlusion of coronary artery after Bentall procedure [6]-[9]. The non-graft related surgical complications include pleural and pericardial effusion [10]-[17], sternal and mediastinal infections [16]-[19], mediastinal hematomas [18] [19], and pulmonary embolism [3]-[20]. Many authors have demonstrated that CT is an invaluable tool in the assessment of post-operative complications after CABG as it is accurate, reproducible, short time, but with a diagnostic level of radiation dose to the patient [1]-[9].

The aim of our present study is to form the basis of a pictorial review on the role of 64-slice HD MDCT in detecting post CABG cardiothoracic complications in our group of patients scanned at our center in clinical radiology department Hospital in Kuwait City.

\section{Materials \& Methods}

\subsection{Patients}

During the period from November 2012 to December 2013, a total of 40 patients with suspected post-CABG cardiothoracic complications underwent MDCT coronary angiography in the clinical radiology department in our Co-Operative Union Cardiac hospital in Kuwait. Informed consent was obtained from all patients and the study had institutional review board approval.

\subsection{MDCT Examination Protocol}

A combination of oral and intravenous beta-blockers has been used prior to the examination. Patients with heart rate $>70$ beat/min received beta-blocker, $100 \mathrm{mg}$ Atenolol (Tenormin, AstraZeneca, Wedel, Germany) orally 1 hour before the scan. If heart rate was still $>70$ beat/min $5 \mathrm{mg}$, metaprolol (Beloc, Astra Zenica, Wedl, Germany) was administered intravenously [6]. No adverse reactions to beta-blocker were recorded.

All examinations were performed by using a 64-slice MDCT scanner (GE Discovery CT750 HD, GE Medical Systems, Milwaukee, USA). Monitoring of the electrocardiogram was performed continuously during the examination, and all image acquisitions were made during a single inspiratory breath-hold. An anterio-posterior and lateral scout acquisitions from clavicle level to the diaphragmatic face of the heart were obtained in each patient to determine the position of the heart and define the scan volume for further imaging. The scan parameters were: $32 \times 0.6 \mathrm{~mm}$ collimation, rotation time $330 \mathrm{~ms}$, table feed $3.8 \mathrm{~mm} /$ rotation; tube voltage $120 \mathrm{kV}$; efficient mA 770 .

For all examinations, 130ml of iodinated contrast agent (Iodixanol, Visipaque $320 \mathrm{mgI} / \mathrm{mL}$, GE Healthcare, Milwaukee, USA) was injected through an 18-gauge cannula positioned in the antecubital vein at a flow rate of $5 \mathrm{ml} / \mathrm{s}$. Before the scan, each patient's individual bolus transit time was determined in the lumen of the ascending aorta using $30 \mathrm{ml}$ intravenous contrast agent at a flow rate of $5 \mathrm{ml} / \mathrm{s}$. The time interval between the bolus injection and the maximal enhancement was measured, and the starting time of the scan was calculated as 3 seconds after the transit time of contrast agent. Smart prep was used in some cases with suspected pulmonary embolism and in patients with arrhythmia. 
For all patients, the standard built-in reconstruction algorithm was used for image reconstruction. The data sets were reconstructed with small field of view (FOV)confined at the heart and with a slice thickness of 0.625 $\mathrm{mm}$ and $0.40 \mathrm{~mm}$ increments during the mid to-end diastolic phase, $65 \%$ to $75 \%$ of the R-R interval. A second dataset was reconstructed with a large FOV to include the entire chest in each cross section with a slice thickness of $5 \mathrm{~mm}$.

All acquired data were transferred to a separate computer workstation (Advanced workstation 4.2, GE Healthcare, Milwaukee, USA) equipped with CardIQ software (GE Medical Systems). Depending on vessel morphology and quality of the MDCT data set, different post-processing techniques such as maximum-intensity projection (MIP), curved multiplanar reconstruction (CMPR), and volume rendering (VR) were applied to assess the origin and course of the coronary vessels. High definition mode was used in all the scans. Gated coronary CT angiography software (Snap-shot Pulse, GE Health care, Milwaukee, USA) was used in 5 patients. Padding of the tube-on time was used to allow the reconstruction to adapt to minor heart rate variation. Patients were scanned with maximal padding of $200 \mathrm{~ms}$ in high-definition mode [9]. The CT examination was performed by an experienced radiologic technologist (A.M.A.). The interpretation of CT images was performed by a senior radiologist (I.M.H.) with an experience of over 15 years exclusively in cardiovascular imaging.

\subsection{Statistical Analysis}

Results were collated and entered into a database (Microsoft Excel 2003) detailing patient characteristics, graft complications, and patient complaints. The data was analysed using statistical package for social science (SPSS) version 21 for windows. The patients' characteristics, graft complications, and complaints were analyzed using descriptive statistics documenting their number.

\section{Results}

Forty patients constituted the study group. There were 35 males and 5 females, their ages ranging from 39 to 60 years with mean age \pm (SD) of $57 \pm 3.9$ years. Young study population is due to a multifactorial risk factors. Patients demographics and characteristics are summarized in Table 1. Four out of 40 patients developed mild allergic reactions such as nausea, vomiting, and erythema but they managed to complete the scan. All patients had a heart rate of 50 to 60 beats/minute at the time of scan acquisition.

A total of 60 grafts were available for evaluation. There were 20 arterial and 40 venous grafts. Graft characteristics of these 40 patients are summarized in Table 2. The patients' complaints are summarized in Table 3.

\begin{tabular}{cc|}
\hline Table 1. Patient characteristics. & \\
\hline Item & Characteristic \\
\hline Number & 40 \\
Gender Females/Males & $5 / 35$ \\
Age (years, Mean \pm SD) & $57 \pm 3.9$ \\
Time postoperative symptoms (days, Mean \pm SD) & $32 \pm 13$ \\
Diabetes & 15 \\
Hypertension & 30 \\
Hypercholesterolemia & 29 \\
Increased serum triglyceride & 20 \\
Atherosclerosis & 18 \\
Obesity & 10 \\
Smoking & 15 \\
Previous myocardial infarction & 1 \\
\hline
\end{tabular}


Table 2. Graft characteristics.

\begin{tabular}{cc}
\hline Graft Characteristic & Number of Grafts \\
Number of grafts & 60 \\
Single arterial grafts & 12 \\
Sequential arterial grafts & 8 \\
Single venous grafts & 22 \\
Sequential venous grafts & 18 \\
Total number of nongrafted vessels & 12 \\
Left anterior descending coronary artery (LAD) & 2 \\
Left circumflex coronary artery (LCx) & 3 \\
Right coronary artery (RCA) & 7 \\
\hline
\end{tabular}

Table 3. Patient complaints.

\begin{tabular}{cc}
\hline Patient Complaint & Number of Patients \\
\hline Chest pain & 40 \\
Respiratory distress & 40 \\
Fever & 7 \\
Dry cough & 2 \\
Productive cough & 2 \\
\hline
\end{tabular}

Two patients could not be evaluated due to clip-artifacts and advanced atherosclerotic disease. Nine patients had graft occlusion at proximal anastomosis and 2 patients at distal anastomosis. Seven patients showed pericardial effusion and 8 patients pleural effusion (Figure 1(a) and Figure 1(b)) diagnosed earlier by echocardiography and chest X-ray. Two patients had chest wall infection by clinical examination, the diagnosis and extent (retrosternal extension and associated mediastinal involvement) of which was confirmed by CT (Figure 2(a) and Figure 2(b)). One patient showed aneurysm at the re-implanted coronary artery after Bentall procedure (Figure 3(a) and Figure 3(b)). One patient showed postoperative myocardial infarction and1 patient dissection of ascending aorta (Figure 4). Another patient showed pseudoaneurysm of left ventricle, previous echocardiography revealed the possibility of diagnosis, but CT confirmed the findings and determined type and extent of the aneurysm (Figures 5(a)-(c)). Two patients had pericardial hematoma which was missed by echocardiography, 2 patients showed pulmonary embolism, and 2 patients showed pneumonia where the plain chest X-ray was normal.

\section{Discussion}

This study was undertaken to form the basis of a pictorial review on the role of CT in detecting post CABG cardiothoracic complications. In our study all grafted and non-grafted vessels were visually classified as abnormal if they showed $\geq 50 \%$ luminal narrowing and/or vessel occlusion. MDCTA was used as it is considered quick, reproducible, and less invasive than other procedures especially in high risk patients where the referring surgeons or treating physicians need clinical answers before treating the patient. Stein PD, et al. and Hausleiter J, et al. [4] [5] showed the accuracy and sensitivity of CTA in the detection of graft stenosis/occlusion. They identified difficulties in visualizing grafted vessel lumen, including: venous against arterial graft, presence of vascular clips which cause artifacts, cardiac motion, atherosclerosis, and site of the graft.

Several authors demonstrated that the diagnostic accuracy of MD CTA in assessment of grafted and nongrafted vessels was considerably lower (73\% to 85\%) [1] whereas other authors found its accuracy to be $100 \%$ [2]-[12]. However, majority of these authors agreed that the accuracy for the evaluation of distal anastomoses is 


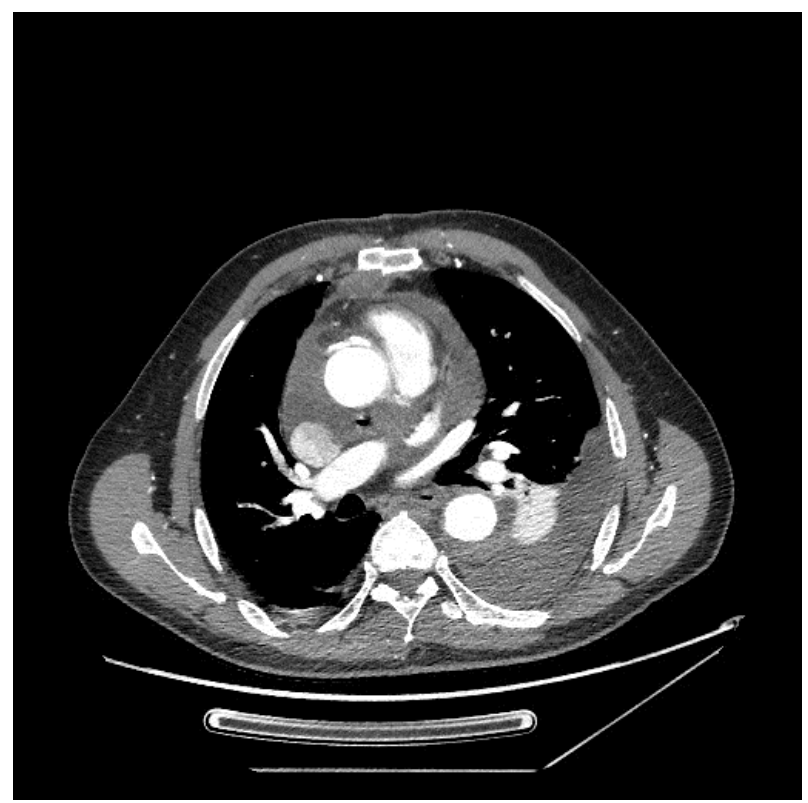

(a)

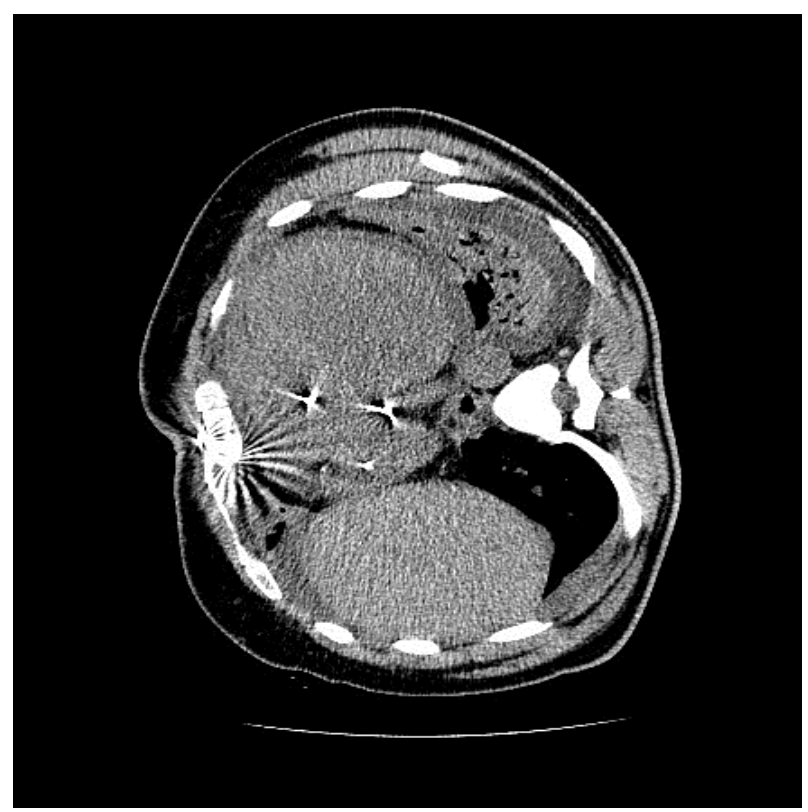

(b)

Figure 1. (a) Axial;and (b) left lateral decubitus axial CT views showing pericardial effusion and pleural effusion.

lower. Metallic artifacts caused by surgical clips may limit the assessment of segments of internal thoracic grafts. Analysis of native vessels is often more difficult in patients who have received coronary artery bypass grafts because of poor distal vessel opacification, more extensive calcification, and smaller diameter lumen size of the grafted vessel.

In our study there were 8 patients with pleural effusion, seven on the left and one in the right side. They were complained of chest pain, heaviness and fullness. One study mentioned that pleural effusion is common after cardiac surgery particularly on the left side, with patients complaining from chest pain or heaviness, shortness of breath and hypoxia [13]. Moreover, pulmonary embolism was presented in two of our patients and these patients complained from chest pain, respiratory distress and were categorized as grade III and IV of respiratory 


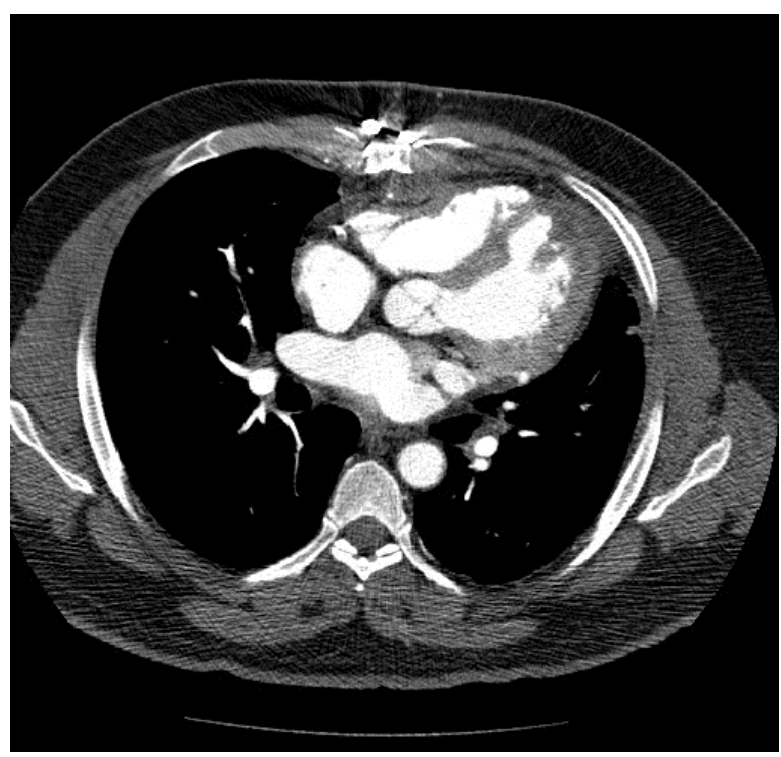

(a)

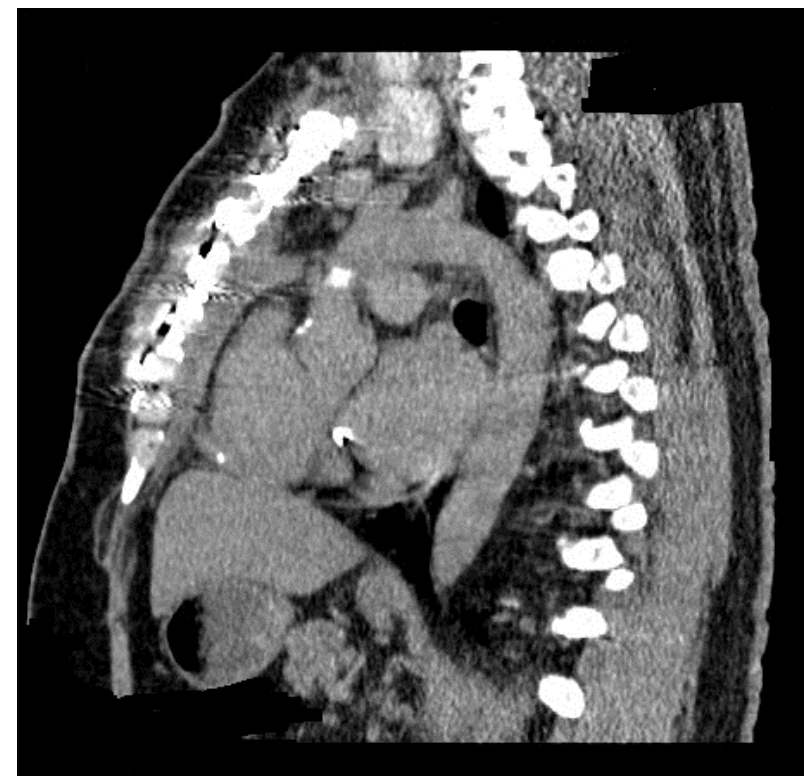

(b)

Figure 2. (a) CT axial view showing postoperative sternal infection; and (b) reconstructed sagittal reformatted image showing air locules and retrosternal fluid collection.

distress. It is reported that the incidence of pulmonary embolism ranges from $0.5 \%$ to $3.5 \%$ and these patients were complained from shortness of breath and/or reduced exercise tolerance [14]. Also in our present study there were two patients who developed pneumonia and presented with productive cough and low grade fever. An author mentioned that most common cause of pneumonia is ventilator associated pneumonia and patients complained of cough, expectoration, and fever [13]. There were 15 patients found to be smokers in our group of patients. It is reported that smoking patients have a higher incidence of pneumonia [14].

There were seven patients complaining of low grade fever due to post-pericardiotomy syndrome and pneumonia and one patient developed high grade fever due to chest wall infection with presence of fistulous tract. It was reported by Zain LK et al. [14] that the noninfectious causes of fever in postoperative open heart surgery is myocardial infarction, post-pericardiotomy syndrome and drug induced fever. However, the infectious causes 


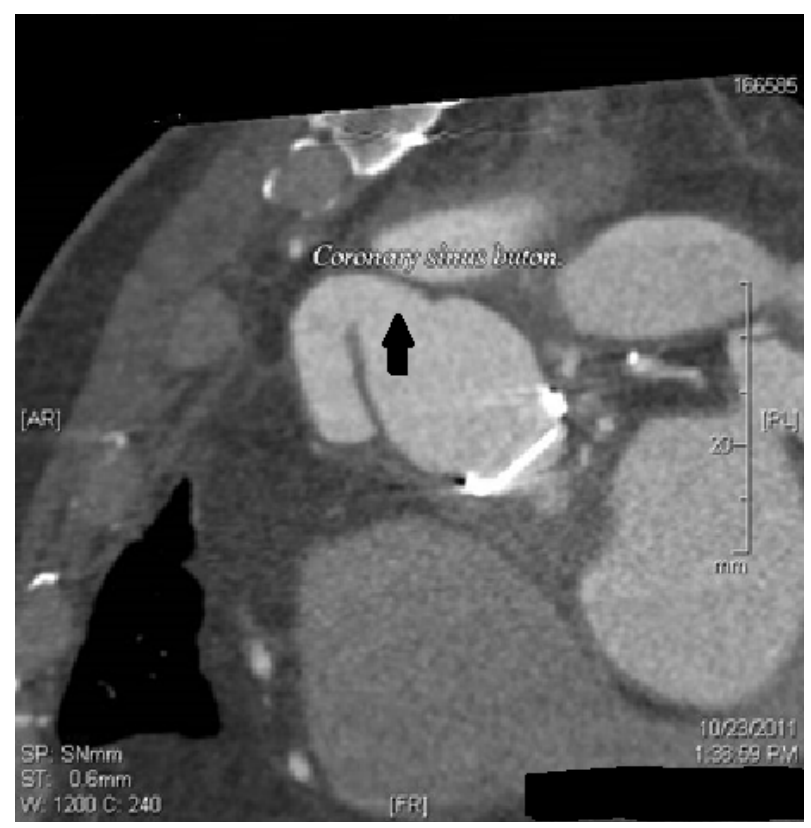

(a)

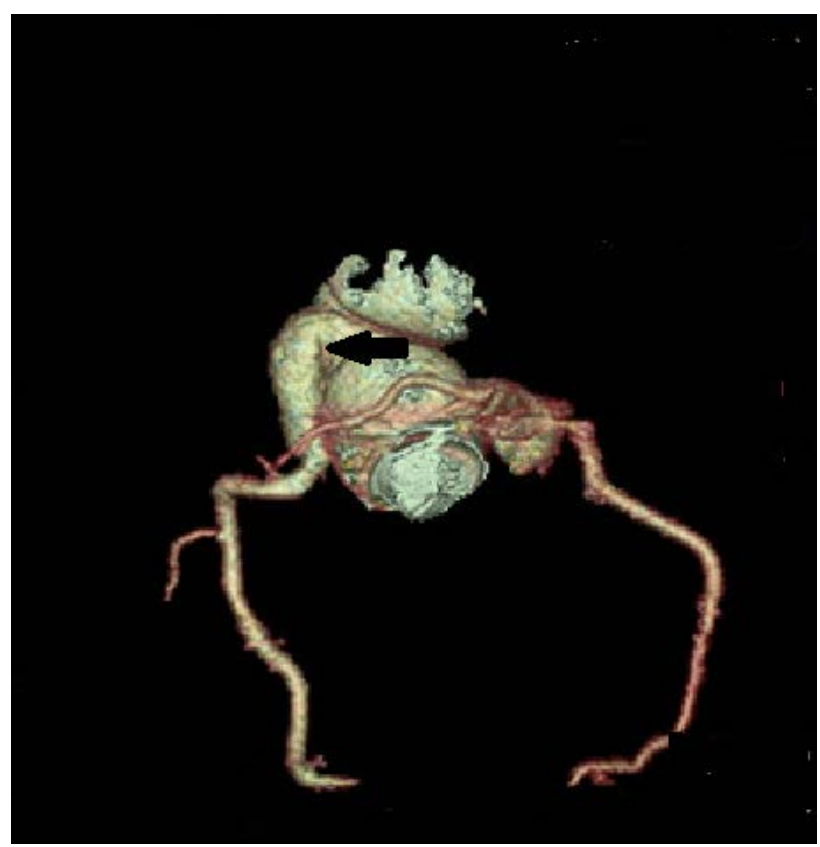

(b)

Figure 3. (a) CT axial view showing aneurysmal dilatation of proximal segment of right coronary artery (arrow); and (b) 3-D reconstructed image after Bental operation (arrow).

include wound infection, urinary tract infection, pneumonia, catheter sepsis, and loculated areas of contaminated blood accumulation in pericardial, pleural, retroperitoneal and leg wound spaces. In the present study there were two patients with chest wall infection, one patient had superficial wound infection but the other patient developed deep sternal wound infection with sinus formation. Durham and Gold [15] stated that the incidence of deep sternal wound infection varies from 0.4 to 5\%. Shirani and Soleymanzadeh [21] mentioned that obesity, diabetes and bilateral internal mammary grafting increase the risk of sternal infection. Fifteen of our patients in the present study were diabetic. 


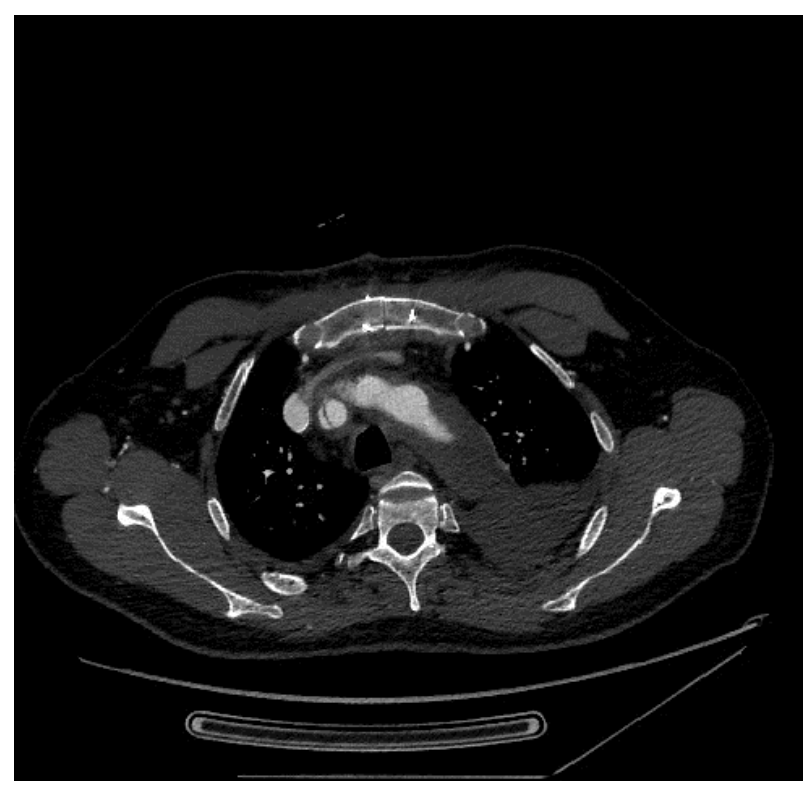

Figure 4. CT axial view showing dissection of the ascending aorta with intimal flap post-CABG and left pleural effusion.

Two patients from the study group had localized pericardial hematoma post Bentall procedure and following CABG operation. Kapoor et al. [16] reported a case of delayed complications related to retained epicardial pacing wires left in situ with consequent organized pericardial hematoma around the wires presenting three months following aortic valve replacement surgery especially in patients on long term oral anticoagulants. Also there were seven patients with pericardial effusion. Ashikhmina AE et al. [17] \& Floerchinger B et al. [18] stated that pericardial effusion is common in patients after open heart surgery due to postoperative bleeding or post-cardiotomy syndrome. They stated that up to $60 \%$ patients often presented with pericardial effusion. They concluded that majority of pericardial effusions after cardiac surgery were localized and frequently located in posterior pericardium or near the right atrium and ventricle with limited accessibility by echocardiography, making them a diagnostic challenge. Diagnostic accuracy of echocardiography is limited in patients after open heart surgery, therefore MDCT is a reasonable alternative for supplemental diagnostic imaging to assess suspected delayed cardiac tamponade in high risk patients after open heart surgery.

In our study there was one patient found to have pseudoaneurysm of left ventricle (Figure 5) whose echocardiography had raised the possibility of diagnosis. Mao C et al. [19] and Makkuni P et al. [20] mentioned that pseudoaneurysm of left ventricle originated form basal inferio-posterior wall of the left ventricle, its incidence is rare andits cause includes myocardial infarction, cardiac surgery, infectious endocarditis and chest trauma. One patient had aneurysm at the re-implanted coronary artery after Bentall procedure. MeherwalZS et al. [22] mentioned that late complications of the Bentall procedure were false or true aneurysm of the arterial button, coronary artery or both.

There were 10 obese patients in this study and they developed more than one complications. Demir A et al. [23] mentioned that obesity does not increase short term mortality for open heart surgery, however, it increases the risk of postoperative pulmonary complications and discharge with morbidity.

ECG gated cardiac MDCT is invaluable following CABG, detailed anatomical information of the heart chambers, pericardium and accurate dynamic evaluation of myocardial function, perfusion and integrity as well as noninvasiveness for diagnosis of postoperative cardiothoracic complications following open heart surgery and it is an excellent, quick, and safe techniques for follow-up. The future of HD MDCT is promising especially in the field of detecting coronary artery disease. One of the limitations of our study is the sample size, and variations of age and sex. This group might not represent the overall population but this data formed the basis of a pictorial review on the role of CT in detecting post CABG cardiothoracic complications. Further studies are needed in larger patients' samples to explore the value of 64-slice HD MDCT in detecting postoperative CABG cardiothoracic surgery complications. Also further studies are needed to compare between HD MDCT and other 


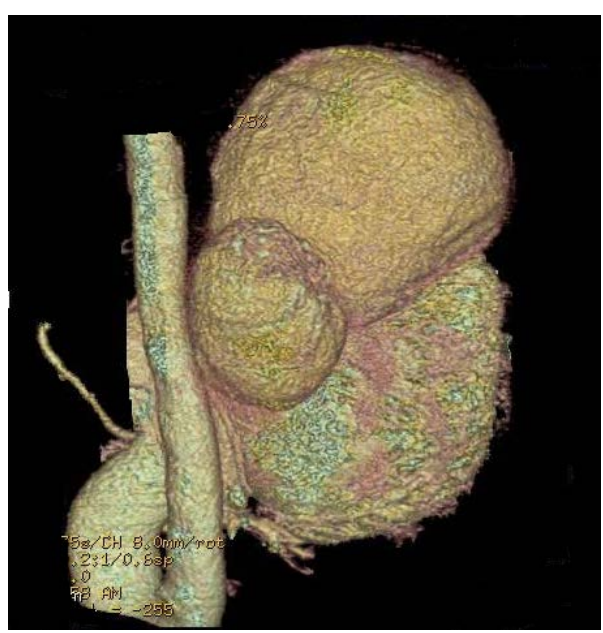

(a)

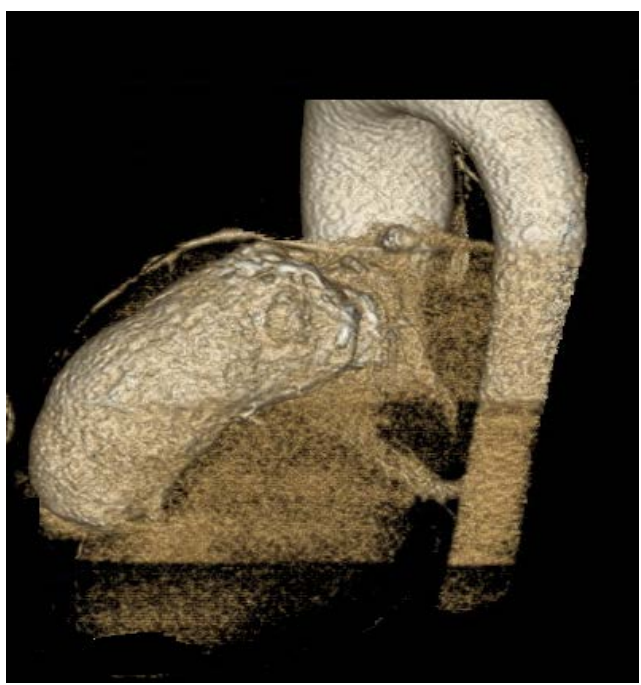

(b)

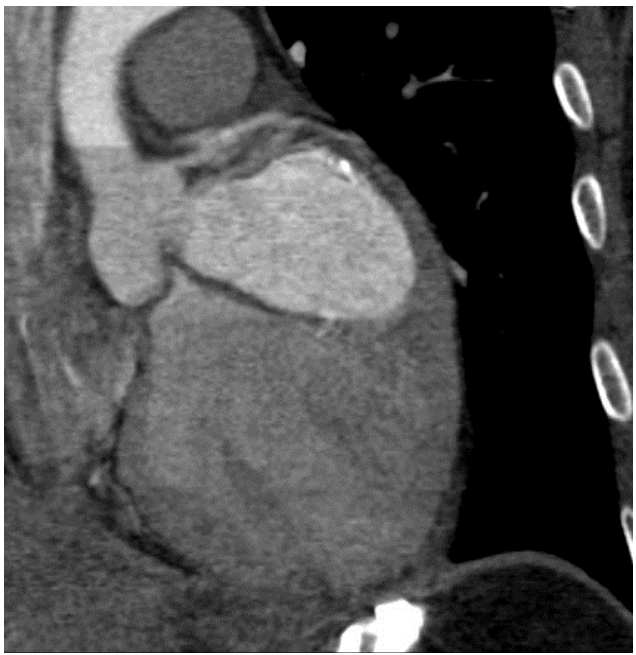

(c)

Figure 5. (a)-(c) 3-D reconstructed CT views showing left ventricular pseudoaneurysm after repair with spared coronary ostium. 
imaging modalities such as coronary angiography and/or magnetic resonance imaging (MRI) and magnetic resonance angiography (MRA) in detecting post-operative cardiothoracic complications following CABG surgery.

\section{Conclusion}

HD MDCT is an easy non-invasive technique which showed to be effective in diagnosis of most postoperative CABG cardiothoracic complications and is gaining more ground in difficult clinical situations.

\section{References}

[1] de Graaf, F.R., van Velzen, J.E., Witkowska, A.J., et al. (2011) Diagnostic Performance of 320-Slicemultidetector Computed Tomography Coronary Angiography in Patients after Coronary Artery Bypass Grafting. European Radiology, 21, 2285-2296. http://dx.doi.org/10.1007/s00330-011-2192-5

[2] Weustink, A.C., Nieman, K. and Pugliese, F. (2009) Diagnostic Accuracy of Computed Tomography Angiography in Patients after Bypass Grafting: Comparison with Invasive Coronary Angiography. Journal of the American College of Cardiology, 2, 816-824.

[3] Türkvatan, A., Bıyıkoğlu, Ş.F., üyükbayraktar, F.G., et al. (2009) Noninvasive Evaluation of Coronary Artery Bypass Grafts and Native Coronary Arteries: Is 16-Slice Multidetector CT Useful? Diagnostic and Interval Radiology, 15, 4350.

[4] Stein, P.D., Beemath, A., Skaf, E., et al. (2005) Usefulness of 4-, 8, and 16-Slice Computed Tomography for Detection of Graft Occlusion or Patency after Coronary Artery Bypass Grafting. American Journal of Cardiology, 96, 16691673. http://dx.doi.org/10.1016/j.amjcard.2005.07.089

[5] Hausleiter, J., Meyer, T., Hadamitzky, M., et al. (2006) Radiation Dose Estimates from Cardiac Multislice Computed Tomography in Dailypractice: Impact of Different Scanning Protocols on Effective Dose Estimates. Circulation, 113, 1305-1310. http://dx.doi.org/10.1161/CIRCULATIONAHA.105.602490

[6] Aviram, G., Mohr, R., Sharony, R., Medalion, B., Kramer, A. and Uretzky, G. (2009) Open Heart Reoperations after Coronary Artery Bypass Grafting: The Role of Preoperative Imaging with Multidetector Computed Tomography. Israel Medical Association Journal, 11, 465-469.

[7] Stauder, N.I., Kuttne, A., Schroder, S., et al. (2006) Coronary Artery Bypass Grafts: Assessment of Graft Patency and Native Coronary Artery Lesions Using 16-Slice MDCT. European Radiology, 16, 2512-2520.

[8] Andreini, D., Pontone, G., Ballerini, G., et al. (2007) Bypass Graft and Native Postanastomotic Coronary Artery Patency: Assessment with Computed Tomography. Annals of Thoracic Surgery, 83, 1672-1678. http://dx.doi.org/10.1016/j.athoracsur.2007.01.030

[9] Nazeri, I., Shahabi, P., Tehrai, M., Sharif-Kashani, B. and Nazeri, A. (2009) Assessment of Patients after Coronary Artery Bypass Grafting Using 64-Slice Computed Tomography. American Journal of Cardiology, 103, 667-673. http://dx.doi.org/10.1016/j.amjcard.2008.10.040

[10] Prat-Gonzalez, S., Sanz, J. and Garcia, M.J. (2008) Cardiac CT: Indications and Limitations. Journal of Nuclear Medicine Technology, 36, 18-24. http://dx.doi.org/10.2967/jnmt.107.042424

[11] Malagutti, P., Nieman, K., Meijboom, W.B., et al. (2007) Use of 64-Slice CT in Symptomatic Patients after Coronary Bypass Surgery: Evaluation of Grafts and Coronary Arteries. European Heart Journal, 28, 1879-1885. http://dx.doi.org/10.1093/eurheartj/ehl155

[12] de Feyter, P.J., Krestin, G.P., Cademartiri, F., et al. (2008) Coronary Bypass Graft Imaging Page 166. CT of the Coronary Arteries Book. Chapter 12, 2nd Edition.

[13] Lawrence, H.C. (2008) Cardiac Surgery in the Adult Textbook. 3rd Edition. McGraw Hill Companies, New York, 478481.

[14] Khalpey, Z.L., Ganim, R.B. and Rawn, J.D. (2008) Postoperative Care of Cardiac Surgery Patients Chapter 16. Cardiac Surgery in the Adult Textbook, 479.

[15] Durham, S.J. and Gold, J.P. (2008) Late Complications of Cardiac Surgery. Chapter 19. Cardiac Surgery in the Adult Textbook, 541.

[16] Kapoor, A., Syal, S., Gupta, N. and Gupta, A. (2011) Right Paracardiac Mass Due to Organized Pericardial Hematoma around Retained Epicardial Pacing Wires Following Aortic Valve Replacement. Interactive Cardio Vascular and Thoracic Surgery, 13, 104-106. http://dx.doi.org/10.1510/icvts.2010.264853

[17] Ashikhmina, E.A., Schaff, H.V, Sinak, L.J., Zhuo, L., Dearani, J.A., Suri, R.M., et al. (2010) Pericardial Effusion after Cardiac Surgery: Risk Factors, Patient Profile, and Contemporary Management. Annals of Thoracic Surgery, 89, 112118. 
[18] Floerchinge, B., Camboni, D., Schopka, S., Kolat, P., Hilker, M. and Schmid, C. (2013) Delayed Cardiac Tamponade after Open Heart Surgery Is Supplemental CT Imaging Reasonable? Journal of Cardiothoracic Surgery, 8, 158. http://dx.doi.org/10.1186/1749-8090-8-158

[19] Mao, C., Li, M.F., Kao, Y.C., Cherng, W.J. and Hung, M.J. (2012) Long Term Survival of a Patient with a Symptomatic Left Ventricle Pseudoaneurysm after Acute Myocardial Infarction. Journal of Internal Medicine Taiwan, 23, 442-448.

[20] Makkuni, P., Kotler, M.N. and Figueredo, V.M. (2010) Diverticular and Aneurysmal Structures of the Left Ventricle in Adults. Texas Heart Institute Journal, 37, 699-705.

[21] Shirani, S. and Soleymanzadeh, M. (2010) Spiral CT of Non-Graft Post Cardiac Surgery Complications: A Pictorial Essay. Iran Journal of Radiology, 7, 11-15.

[22] Meherwal, Z.S., Collison, S.P., Gupta, A., Choudhary, A. and Saraf, N. (2011) Aortic Root Replacement in Young Adults: Disease Characteristics and Early Outcome. Indian Journal of Thoracic and Cardiovascular Surgery, 27, 1-6.

[23] Demir, A., Aydlnll, B., Guclu, C.Y., et al. (2012) Obesity and Postoperative Early Complications in Open Heart Surgery. Journal of Anesthesia, 26, 702-710. http://dx.doi.org/10.1007/s00540-012-1393-7 